\title{
Evidence for Expanding Quantum Field Theory
}

\author{
John R. Klauder \\ Department of Physics and Department of Mathematics, University of Florida, Gainesville, USA \\ Email: klauder@ufl.edu
}

How to cite this paper: Klauder, J.R. (2021) Evidence for Expanding Quantum Field Theory. Journal of High Energy Physics, Gravitation and Cosmology, 7, 1157-1160. https://doi.org/10.4236/jhepgc.2021.73067

Received: June 16, 2021

Accepted: July 16, 2021

Published: July 19, 2021

Copyright $\odot 2021$ by author(s) and Scientific Research Publishing Inc. This work is licensed under the Creative Commons Attribution International License (CC BY 4.0)

http://creativecommons.org/licenses/by/4.0/

\begin{abstract}
Present day Quantum Field Theory (QFT) is founded on canonical quantization, which has served quite well but also has led to several issues. The free field describing a free particle (with no interaction term) can suddenly become nonrenormalizable the instant a suitable interaction term appears. For example, using canonical quantization $\varphi_{4}^{4}$, has been deemed a "free" theory with no difference from a truly free field [1] [2]. Using the same model, affine quantization has led to a truly interacting theory [3]. This fact alone asserts that canonical and affine tools of quantization deserve to be open to their procedures together as a significant enlargement of QFT.
\end{abstract}

\section{Keywords}

Quantum Field Theory, Canonical Quantization, Affine Quantization

\section{Introduction}

\subsection{Classical Starting Point}

There are different ways to promote classical to quantum expressions that are useful. For the classical, canonical Hamiltonian model, we have

$$
H(\pi, \varphi)=\int\left\{\frac{1}{2}\left[\pi(x)^{2}+(\vec{\nabla} \varphi(x))^{2}+m^{2} \varphi(x)^{2}\right]+g \varphi(x)^{r}\right\} \mathrm{d}^{s} x,
$$

where $r \geq 2$ and $s \geq 3$. The ingredients in this expression are the classical field $\varphi(x)$ and the momentum field $\pi(x)$. These fields obey the Poisson bracket $\{\varphi(x), \pi(y)\}=\delta^{s}(x-y)$.

However, we can describe the same Hamiltonian in a different way. Let us choose the affine field $k(x) \equiv \pi(x) \varphi(x)$, instead of the momentum field, but still keep $\varphi(x)$. However, it is necessary to keep $\varphi(x) \neq 0$ for otherwise if 
$\varphi(x)=0$, then $\pi(x)$ means nothing. Let us exam (1), the same classical Hamiltonian, but now in the new coordinates, leading to

$$
H^{\prime}(\kappa, \varphi)=\int\left\{\frac{1}{2}\left[\kappa(x)^{2} \varphi(x)^{-2}+(\vec{\nabla} \varphi(x))^{2}+m^{2} \varphi(x)^{2}\right]+g \varphi(x)^{r}\right\} \mathrm{d}^{s} x .
$$

This new set of fields leads to the Poisson bracket $\{\varphi(x), \kappa(y)\}=\delta^{s}(x-y) \varphi(x)$.

\subsection{Quantum Starting Point}

Promotion of the fields $\varphi(x) \rightarrow \hat{\varphi}(x)$ and $\pi(x) \rightarrow \hat{\pi}(x)$, leads to the traditional quantum expression for our Hamiltonian, which is given by

$$
H(\hat{\pi}, \hat{\varphi})=\int\left\{\frac{1}{2}\left[\hat{\pi}(x)^{2}+(\vec{\nabla} \hat{\varphi}(x))^{2}+m^{2} \hat{\varphi}(x)^{2}\right]+g \hat{\varphi}(x)^{r}\right\} \mathrm{d}^{s} x .
$$

Now, knowing that the classical variables were no longer the canonical choice but rather the affine coordinates, and after the promotion of affine field variables to new quantum field variables, the new quantum Hamiltonian becomes

$$
H^{\prime}(\hat{\kappa}, \hat{\varphi})=\int\left\{\frac{1}{2}\left[\hat{\kappa}(x) \hat{\varphi}(x)^{-2} \hat{\kappa}(x)+(\vec{\nabla} \hat{\varphi}(x))^{2}+m^{2} \hat{\varphi}(x)^{2}\right]+g \hat{\varphi}(x)^{r}\right\} \mathrm{d}^{s} x .(4)
$$

Do not worry about $\hat{\varphi}(x)^{-2}$ because we have already insisted that $\varphi(x) \neq 0$; hence $\hat{\varphi}(x) \neq 0$. In a previous usage, which proved itself by modifying $\hat{\varphi}(x)^{-2} \rightarrow\left[\hat{\varphi}(x)^{2}+\varepsilon\right]^{-1}$, while $\varepsilon=10^{-10}$ served as a safeguard [4].

It is noteworthy that $\hat{\kappa}(x) \hat{\varphi}(x)^{-1 / 2}=0$, which, in Schrödinger's representation, leads to $\hat{\kappa}(x)=-i \hbar[\varphi(x)(\partial / \partial \varphi(x))+(\partial / \partial \varphi(x)) \varphi(x)] / 2$ and $\hat{\varphi}(x)=\varphi(x)$. Following a suitable regularization process [5], this yields the stated result.

\subsection{Advantages of an Affine Quantization}

Using the results of the previous sections we propose that $\hat{\kappa}(x) \Pi_{y} \varphi(y)^{-1 / 2}=0$ which exposes our choice for general wave functions as given by $\Psi(\varphi)=\int W(\varphi(x)) \Pi_{y} \varphi(y)^{-1 / 2} \mathrm{~d} \varphi(x)$. A regularized version, using $x \rightarrow \mathbf{k}$ where $\mathbf{k}=a n=a \times\{\cdots,-2,-1,0,1, \cdots\}^{s}$ of this expression looks like $\Psi(\varphi)=w(\varphi) \Pi_{\mathbf{k}}\left(b a^{s}\right)^{1 / 2}\left(\varphi_{\mathbf{k}}\right)^{-\left(1-2 b a^{s}\right) / 2}$, where $b a^{s}$ is dimensionless and $b \sim 1$.

We now take a Fourier transformation of the absolute square of our regulated wave function that looks like

$$
F(f)=\Pi_{\mathbf{k}} \int\left\{\mathrm{e}^{i f_{\mathbf{k}} \varphi_{\mathbf{k}}}\left|w\left(\varphi_{\mathbf{k}}\right)\right|^{2}\left(b a^{s}\right)\left|\varphi_{\mathbf{k}}\right|^{-\left(1-2 b a^{s}\right)} \mathrm{d} \varphi_{\mathbf{k}}\right\} .
$$

Normalization ensures that if all $f_{\mathbf{k}}=0$, then $F(0)=1$, which leads to

$$
F(f)=\Pi_{\mathbf{k}} \int\left\{1-\int\left(1-\mathrm{e}^{i f_{\mathbf{k}} \varphi_{\mathbf{k}}}\right)\left|w\left(\varphi_{\mathbf{k}}\right)\right|^{2}\left(b a^{s}\right) \mathrm{d} \varphi_{\mathbf{k}} /\left|\varphi_{\mathbf{k}}\right|^{\left(1-2 b a^{s}\right)}\right\} .
$$

Now, at last, we can let $a \rightarrow 0$ to fix the Fourier transformation ${ }^{1}$

${ }^{1}$ Any change of $w(\varphi)$ due to $a \rightarrow 0$ is left implicit. 


$$
F(f)=\exp \left\{-b \int \mathrm{d}^{s} x\left(1-\mathrm{e}^{i f(x) \varphi(x)}\right)|w(\varphi(x))|^{2} \mathrm{~d} \varphi(x) /|\varphi(x)|\right\} .
$$

Observe that the affine quantization his led to a Poisson distribution, which is the only other term, besides a Gaussian expression, as dictated by The Central Limit Theorem [6]. Nevertheless, the same expression as in (7) could have arisen when $g=0$, or even when $g \nearrow \searrow 0$, asserting that our final result is definitely not a Gaussian! Of significant is the fact that if the coupling $g$, or even the mass $m$, are smoothly changed, there are only continuous changes within $w(\varphi)$. Also, the fact that $\hat{\kappa}(x) \varphi(x)^{-1 / 2}=0$, which is a dramatic change from canonical theory's equivalent relation, i.e., $\hat{\pi}(x) \mathbb{1}=0$, makes a big difference; indeed, the factor $\left|\varphi_{\mathbf{k}}\right|^{-\left(1-2 b a^{s}\right)}$ in (5) is the key to avoiding a Gaussian result. Apparently, this behavior of affine quantization adopts the least final domain at the outset, which overcomes any threat of nonrenormalizability. ${ }^{2}$

\section{Summary}

We have obtained a continuous, fully regularized, expression that implicitly involves a large sample of quantum field models. The application of affine quantization, but not canonical quantization, has offered us a treasure of interest that presently rests in the Fourier representation space. Understanding the physics needed to clarify our results requires a second Fourier transformation back into the original space of the classical field, here given by $\varphi(x)$. That issue is purely a mathematical task, and the implications of such an effort are certainly of great interest!

\section{Conflicts of Interest}

The author declares no conflicts of interest regarding the publication of this paper.

\section{References}

[1] Aizenman, M. (1981) Proof of the Triviality of $\varphi_{d}^{4}$ Field Theory and Some Mean-Field Features of Ising Models for $d>4$. Physical Review Letters, 47, 1-4, E-886. https://doi.org/10.1103/PhysRevLett.47.886

[2] Fröhlich, J. (1982) On the Triviality of $\lambda \varphi_{d}^{4}$ Theories and the Approach to the Critical Point in $d \geq 4$ Dimensions. Nuclear Physics B, 200, 281-296. https://doi.org/10.4007/annals.2021.194.1.3

[3] Fantoni, R. and Klauder, J.R. (2021) Affine Quantization of $\varphi_{4}^{4}$ Succeeds, While Canonical Quantization Fails. Physical Review D, 103, Article ID: 076013. https://doi.org/10.1103/PhysRevD.103.076013

[4] Fantoni, R. (year) Monte Carlo Evaluation of the Continuum Limit of $\varphi_{3}^{12}$. https://arxiv.org/abs/2011.09862

[5] Klauder, J.R. (2020) Using Affine Quantization to Analyze Non-Renormalizable ${ }^{2}$ For those who wish to learn more about affine quantization see [5]. For beginners, canonical quantization deals with the harmonic oscillator, but the half-harmonic oscillator requires affine quantization [7]. 
Scalar Fields and the Quantization of Einstein's Gravity. Journal of High Energy Physics, Gravitation and Cosmology, 6, 802-816.

https://doi.org/10.4236/jhepgc.2020.64053

[6] Central Limit Theorem. Wikipedia.

https://en.wikipedia.org/wiki/Category:Central_limit_theorem

[7] Gouba, L. (2021) Affine Quantization on the Half Line. Journal of High Energy Physics, Gravitation and Cosmology, 7, 352-365.

https://doi.org/10.4236/jhepgc.2021.71019 